\title{
Geographical Differences in Dietary Exposure to Perfluoroalkyl Acids between Manufacturing and Application Regions in China
}

\author{
Haiyan Zhang, ${ }^{\dagger,}$ Robin Vestergren, ${ }^{\S}, \|$ Thanh Wang, ${ }^{*, \perp, \ddagger \odot}$ Junchao Yu, ${ }^{\ddagger}$ Guibin Jiang, ${ }^{\ddagger}$
} and Dorte Herzke*,\$(0)

${ }^{\dagger}$ College of Environment, Zhejiang University of Technology, Hangzhou 310032, China

${ }^{\ddagger}$ State Key Laboratory of Environmental Chemistry and Ecotoxicology, Research Center for Eco-Environmental Sciences, Chinese Academy of Sciences, Beijing 100085, China

${ }^{\S}$ Norwegian Institute for Air Research (NILU), FRAM - High North Research Centre on Climate and the Environment, Tromsø, Norway

"ACES - Department of Environmental Science and Analytical Chemistry, Stockholm University, Stockholm, Sweden

${ }^{\perp}$ MTM Research Centre, School of Science and Technology, Örebro University, Örebro, Sweden

\section{Supporting Information}

\begin{abstract}
Emissions of perfluoroalkyl acids (PFAAs) have increased in China over the past decade, but human exposure pathways are poorly understood. Here we analyzed 15 PFAAs in commonly consumed food items and calculated body weight normalized dietary intake rates (estimated dietary intake, EDIs) in an area with ongoing PFAA production (Hubei province; $n=121$ ) and an urbanized coastal area (Zhejiang province; $n=106)$. Geographical differences in concentrations were primarily observed for perfluorooctanesulfonic acid (PFOS) and perfluorohexanesulfonic acid (PFHxS) in animal food items and shortchain PFAAs in vegetable food items. The average EDI of $\sum$ PFAAs for adults in Hubei (998 $\mathrm{ng} \mathrm{kg}^{-1} \mathrm{day}^{-1}$ ) was more than 2 orders of magnitude higher than that in Zhejiang $\left(9.03 \mathrm{ng} \mathrm{kg}^{-1} \mathrm{day}^{-1}\right)$. In Hubei province, the average EDI of PFOS for adults $\left(87 \mathrm{ng} \mathrm{kg}^{-1}\right.$ day $\left.{ }^{-1}\right)$ was close to or exceeded advisory guidelines used in other countries indicating health risks for the population from long-term exposure. Yet, PFOS could only account for about $10 \%$ of the EDI of $\sum$ PFAAs in the Hubei province, which was dominated by short-chain PFAAs through consumption of vegetables. The large contribution of short-chain PFAAs to the total EDIs in manufacturing areas emphasize the need for improved exposure and hazard assessment tools of these substances.
\end{abstract}

\section{INTRODUCTION}

Perfluoroalkyl acids (PFAAs) are a commercially important group of synthetic chemicals that contain a fully fluorinated carbon chain and an acid headgroup which is most commonly sulfonic acid (PFSA) or carboxylic acid (PFCA). ${ }^{1}$ The combination of the perfluoroalkyl moiety and acidic functional group gives PFAAs unique surfactant properties and chemical stability which is useful in many industrial applications. ${ }^{1}$ Although PFAAs have been produced in increasing quantities since the 1950s, it was only after the discoveries of perfluorooctanesulfonic acid (PFOS) in humans and wild-life that scientists and regulators started paying attention to their problematic environmental properties. ${ }^{2}$ Long-chain PFSAs $\left(\mathrm{C}_{n} \mathrm{~F}_{2 n+1} \mathrm{SO}_{3} \mathrm{H}, n \geq 6\right)$ and PFCAs $\left(\mathrm{C}_{n} \mathrm{~F}_{2 n+1} \mathrm{COOH}, n \geq 7\right)$ are of particular concern due to their environmental persistence, bioaccumulation potential and toxicity. ${ }^{3}$

Increased public awareness and stricter regulations in Europe and North America have led to a number of changes in PFAA production and use globally. In 2000-2002, the major global manufacturer of PFOS and related perfluorooctane sulfonyl fluoride (POSF) derivatives ceased production of these chemicals. More recently, a phase-out strategy of perfluorooctanoic acid (PFOA) and related telomer-based derivatives was implemented by eight leading PFAA producing companies. ${ }^{4}$ These phase-out actions have been partly accomplished by substituting long-chain PFAAs, such as PFOS and PFOA, with a variety of fluorinated alternatives which are typically shorter chain versions of their predecessors or per- or polyfluorether compounds. ${ }^{5}$ The rationale for promoting the replacements is the lower bioaccumulation potential in aquatic organisms and more rapid elimination in mammalian species. $^{6-10}$ However, it remains widely debated whether or not these substances can be considered as safe alternatives. ${ }^{11,12}$

Received: January 13, 2017

Revised: April 5, 2017

Accepted: April 7, 2017

Published: April 7, 2017 
The limited data available for per- and polyfluoroether acids suggest that they have a similar bioaccumulation as their corresponding PFAAs. ${ }^{13,14}$ For short-chain PFAAs, on the other hand, it remains questionable if the lower bioaccumulation potential is sufficient to protect human populations from an elevated exposure. Another important trend in the production and use of PFAAs is the continuous use of longchain PFAAs in emerging economies such as China. ${ }^{15,16}$ An increasing number of studies have recently reported on the emissions of both legacy and replacement PFAAs from different parts of China, ${ }^{17-19}$ but the impact of these emissions on human exposure remains poorly understood.

Exposure assessments from Europe and North America have identified dietary intake to be a major exposure pathway of PFOA and PFOS for the general population. ${ }^{20-22}$ However, the data sets on PFAAs in food items from China remain rather limited with most studies focusing on animal and dairy products. ${ }^{23-27}$ In contrast to the typical western diet, the traditional Chinese diet is usually low in animal fat and high in dietary fiber with vegetables accounting for more than half of the dietary intake on a mass basis. ${ }^{28,29}$ Thus, consideration of dietary intake of PFAAs via vegetables may be particularly important in China compared to western countries. Considering the numerous ongoing point sources of PFAAs and varying dietary habits, there may also be large geographical differences in dietary exposure to PFAAs within China.

In this paper, we provide one of the most comprehensive dietary intake assessments of PFAAs from China to date. Specific emphasis was placed on elucidating geographical differences between PFAA manufacturing and application areas and quantifying dietary exposure pathways for shortchain PFAAs. A large number of locally produced food items were collected from Hubei province $(n=121)$, and Zhejiang province $(n=106)$. The samples were analyzed for 15 perfluoroalkyl acids as well as perfluorooctane sulfonamide (PFOSA), and combined with regional food consumption statistics to estimate the total dietary intake.

\section{EXPERIMENTAL SECTION}

2.1. Sample Collection. Hubei and Zhejiang province in China were selected for this sampling campaign due to their difference in production and use patterns (Figure 1). Hubei is the major province for production of PFOS and related chemicals in China. ${ }^{15}$ The sampling area from Hubei included one of the largest facilities of PFOS and POSF-derivatives in China. In contrast, Zhejiang province has little documented production of PFAAs. As a densely populated and highly industrialized coastal province there are, however, multiple potential applications of PFAAs in textile and leather treatment, metal plating, fluoropolymer manufacture and fire-fighting foams at airports (e.g., Xiaoshan Airport which is marked in Figure 1). ${ }^{15,30}$ Emission inventories of PFOA and PFOS further suggest that diffuse emissions are relatively more important in Zhejiang compared to Hubei province. ${ }^{15,31}$

A total of 227 samples of commonly consumed food items were collected in the two provinces during the period of September to November 2012. More than 20 different food types of plant origin were included and grouped into 4 different food categories; cereals $(n=9)$, tubers $(n=8)$, legumes $(n=$ $13)$, and other leafy vegetables $(n=100)$. Food items of animal origin included livestock meat $(n=6)$, poultry meat $(n=30)$, offal (edible livers of pork, duck and chicken, $n=22)$, eggs $(n=$ $14)$, fish $(n=18)$, and fish liver (not commonly consumed in
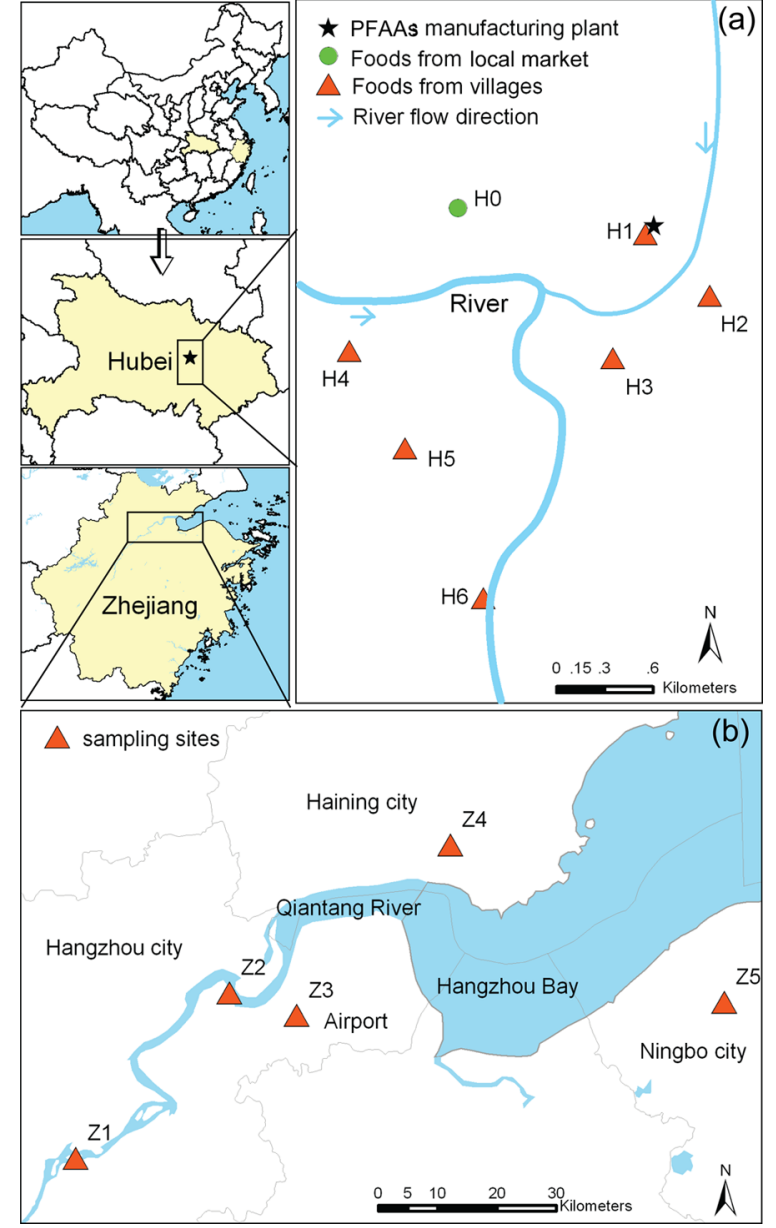

Figure 1. Sampling sites in (a) Hubei and (b) Zhejiang provinces.

China, $n=7$ ) (for further details, see Table S1 in the Supporting Information (SI)). Most of the food samples were directly collected from the households and farms of local residents. Crops were washed with tap water to remove dust or soil from the surface. Free range chicken and ducks were purchased from the local residents and sacrificed on place. Their meat and liver was removed and wrapped in aluminum foil. Livestock meat (pork and beef) and pork liver were purchased from local markets in the villages. Fish samples were captured from rivers near the villages or purchased from the local market. All the samples were wrapped in aluminum foil, placed into different plastic bags, and then transported to the laboratory. Only edible parts of all samples (after peeling off or cutting the roots) were homogenized using a kitchen blender and thereafter freeze-dried, then stored in a fridge at $-20{ }^{\circ} \mathrm{C}$ until sample pretreatment and analysis.

2.2. Chemicals and Materials. All standards were purchased from Wellington Laboratories (Guelph, ON, Canada). The 16 analytes included 4 PFSAs (PFBS, PFHxS, branched and linear PFOS (brPFOS, linPFOS), perfluorodecanesulfonic acid (PFDcS)), 11 PFCAs (perfluorobutanoic acid (PFBA), perfluoropentanoic acid (PFPA), perfluorohexanoic acid (PFHxA), perfluoroheptanoic acid (PFHpA), perfluorooctanoic acid (PFOA), perfluorononanoic acid (PFNA), perfluorodecanoic acid (PFDcA), perfluoroundecanoic acid (PFUnDA), perfluorododecanoic acid (PFDoDA), perfluorotridecanoic acid (PFTrDA), perfluorotetradecanoic acid (PFTeDA)), and perfluorooctane sulfonamide (PFOSA). 
Isotope labeled internal standards (IS) included $\left[{ }^{13} \mathrm{C}_{4}\right]$-PFBA, $\left[{ }^{13} \mathrm{C}_{2}\right]$-PFHxA, $\left[{ }^{13} \mathrm{C}_{4}\right]$-PFOA, $\left[{ }^{13} \mathrm{C}_{5}\right]$-PFNA, $\left[{ }^{13} \mathrm{C}_{2}\right]$-PFDcA, $\left[{ }^{13} \mathrm{C}_{2}\right]$-PFUnDA, $\left[{ }^{13} \mathrm{C}_{2}\right]$-PFDoDA, $\left[{ }^{18} \mathrm{O}_{2}\right]$-PFHxS, $\left[{ }^{13} \mathrm{C}_{4}\right]$ PFOS, and $\left[{ }^{13} \mathrm{C}_{8}\right]$-PFOSA were applied as mass-labeled internal standards (IS) (SI Table S2), all donated by Wellington Laboratories (Guelph, Canada), and branched perfluorodecanoic acid (brPFDcA) was used as an injection standard.

All solvents and reagents were of HPLC grade (MerckSchuchardt, Hohenbrunn, Germany). A Milli-Q system (Millipore, Billerica, MA) was used and the generated water was further passed through a mixed mode C8 plus quaternary amine (CUQAX) SPE cartridge. Florisil sorbent (60/100 mesh) and graphitized carbon (Supelclean ENVI-Carb, 120/ 400 mesh) were purchased from Supelco (Bellefonte, PA). Florisil sorbent was dried at $450{ }^{\circ} \mathrm{C}$ overnight and deactivated with HPLC water at $0.5 \%(\mathrm{w} / \mathrm{w})$ before usage.

2.3. Extraction and Clean Up. The freeze-dried samples and field blank samples were transported to Norwegian Institute for Air Research (NILU) in Norway for subsequent pretreatment and analysis. The extraction and cleanup protocol was based on the method described by Vestergren et al. ${ }^{32}$ with some minor modification. In short, approximately $1 \mathrm{~g}$ dry weight of food sample was weighted into a $50 \mathrm{~mL}$ polypropylene (PP) tube. Isotope labeled internal standards $(2.5 \mathrm{ng}$ ) and $6 \mathrm{~mL}$ of $400 \mathrm{mM} \mathrm{NaOH}$ was added and the sample was allowed to equilibrate at $4{ }^{\circ} \mathrm{C}$ overnight. Thereafter, $4 \mathrm{~mL}$ tetrabutyl ammonium hydrogen sulfate (TBA) solution, 8 $\mathrm{mL} 250 \mathrm{mM} \mathrm{Na} \mathrm{CO}_{3} / \mathrm{NaHCO}_{3}$ buffer, and $10 \mathrm{~mL}$ methyl tertbutyl ether (MTBE) were added and the mixture was vortexed for $30 \mathrm{~s}$. The samples were extracted in an ultrasonic bath at room temperature for $10 \mathrm{~min}$ and phase separation was carried out by centrifugation at $3500 \mathrm{rpm}(4110 \mathrm{~g})$ for $10 \mathrm{~min}$. The organic phase was then transferred to a $15 \mathrm{~mL}$ PP tube. The extraction was repeated twice with $5 \mathrm{~mL}$ MTBE for each extraction. The extracts were combined and concentrated to a final volume of approximately $1 \mathrm{~mL}$ using a Rapidvap nitrogen evaporation system (Labconco). A $5 \mathrm{~mL}$ disposable glass pipet with a glass wool plug was used for cleanup, and was filled with $1.5 \mathrm{~g}$ of Florisil mixed with $25 \mathrm{mg}$ of ENVI-carb at the bottom and $1 \mathrm{~g}$ of anhydrous granular $\mathrm{Na}_{2} \mathrm{SO}_{4}$ at the top. The column was rinsed with $5 \mathrm{~mL}$ of methanol $(\mathrm{MeOH})$ and then conditioned with $5 \mathrm{~mL}$ of MTBE. Thereafter, the sample extract was loaded and the column was washed with $10 \mathrm{~mL}$ of MTBE. Subsequently, the target analytes were eluted with 10 $\mathrm{mL}$ of a $30 / 70 \mathrm{MeOH} / \mathrm{MTBE}$ mixture (v/v). The eluate was evaporated to $\sim 500 \mu \mathrm{L}$ using Rapidvap after which $2 \mathrm{ng}$ brPFDcA standard was added. The final solution was stored in a refrigerator until analysis.

2.4. Instrumental Methods. $100 \mu \mathrm{L}$ of the final solution was mixed with $100 \mu \mathrm{L}$ of $2 \mathrm{mM} \mathrm{NH}_{4} \mathrm{OAc}$ for instrumental analysis. The instrumental analysis method for PFAAs was performed by an ultrahigh pressure liquid chromatograph coupled with a triple-quadrupole mass-spectrometer (UHPLC-MS/MS) according to Hanssen et al. ${ }^{33}$ Analysis was performed on a Thermo Scientific Vantage MS/MS (Vantage TSQ) (Thermo Fisher Scientific Inc., Waltham, MA); using a Waters Acquity UPLC HSS $3 \mathrm{~T}$ column $(2.1 \times 100$ $\mathrm{mm}, 1.8 \mu \mathrm{m}$ ) (Waters Corporation, Milford, MA) equipped with a Waters Van guard HSS T3 guard column $(2.1 \times 5 \mathrm{~mm}$, $1.8 \mu \mathrm{m}$ ) (Waters Corporation, Milford, MA). Separation was achieved using $2 \mathrm{mM} \mathrm{NH}_{4} \mathrm{OAc}$ in 90:10 water/MeOH (A) and $2 \mathrm{mM} \mathrm{NH}_{4} \mathrm{OAc}$ in $\mathrm{MeOH}$ (B) as the mobile phases. A Waters XBridge C18 column $(2.1 \times 50 \mathrm{~mm}, 5 \mu \mathrm{m})$ (Waters
Corporation, Milford, MA) was installed after the pump and before the injector. The analytical conditions, parent ions, monitored transitions, collision energies and S-lens are shown in SI Table S3. Quantification was conducted using the LCQuan software from Thermo Scientific (Version 2.6) (Thermo Fisher Scientific Inc., Waltham, MA).

2.5. QA/QC. An internal standard method using isotopic dilution was emplyed to ensure accurate identification and quantification of the analytes. Isotope labeled PFAAs were used for all analytes except PFPA, PFHpA, PFTrDA, PFTeDA, and PFBS. For these analytes the closest isotope labeled PFCAs or PFSAs based on retention time standard was used for quantification. Peaks with a signal-to-noise ratio $(\mathrm{S} / \mathrm{N})>3$ were identified based on the retention time compared with the corresponding standards. Field blanks were deployed at each region by opening a clean polypropylene container filled with anhydrous sodium sulfate at the sampling site for about $2 \mathrm{~h}$. Freeze-drying blanks (anhydrous sodium sulfate added to freeze frying batches) and extraction procedural blanks were used to assess potential field and laboratory contamination. Limit of quantitation (LOQ) was defined as values of the lowest detectable calibration standard corresponding to the peak with $\mathrm{S} / \mathrm{N} \geq 10$. For PFAAs with no detectable blank contamination, LOQ was used to calculate the method limit of detection (MDL). For PFAAs with detectable concentrations in procedural or field blanks; these were used to define the MDL as the arithmetic mean plus three times the standard deviation of blank values. MDLs were in the range $0.01-0.07 \mathrm{ng} / \mathrm{g}$ for most PFAAs and PFOSA, except for brPFOS and PFDcS which had MDLs ranged from 0.14 to $0.33 \mathrm{ng} / \mathrm{g}$. Trace amounts of PFBS and PFBA were found in the field and freeze-drying blanks for Hubei samples, and the MDLs for these two analytes were calculated to 0.23 and $0.17 \mathrm{ng} / \mathrm{g}$, respectively. More details of LOQ and MDL are shown in SI Table S4. The recoveries for surrogate standard of $\left[{ }^{13} \mathrm{C}\right]$-PFAAs ranged from $54 \% \pm 29 \%$ to $96 \% \pm 25 \%$ (SI Table S5). All the results in this study were reported on a wet weight basis and were not blank corrected. Accuracy and precision was evaluated by analyzing a set of reference materials consisting of pig liver, fish muscle and pea homogenate supplied by the KBBE EU project PERFOOD, and compliancy of the currently used methods were previously reported by the authors and published by Weiss et al. ${ }^{34}$ The analytical method utilized by us achieved Z-scores between 0.06 and 1.4 for 12 target PFAAs. ${ }^{35}$ A subset of samples $(n=9)$ with high concentrations of PFBA and PFPA were selected for reanalysis using UPLC coupled with a quadrupole time-of-flight (qTOF) MS at Stockholm University according to the method established by Ullah et al. ${ }^{36}$ to confirm the identification of these analytes by accurate mass since they do not have a qualifier ion in MS/MS. All results confirmed the positive detection and quantification of these PFAA.

2.6. Dietary Intake Calculations. For calculation of total dietary intake the individual food items were grouped into different food categories (as described above). The body weight normalized estimated daily intake of PFAAs (EDI; $\mathrm{ng} \mathrm{kg}^{-1}$ day $^{-1}$ ) was subsequently calculated by the following equation

$$
\mathrm{EDI}=\frac{\sum_{i-1}^{n} C_{\mathrm{food}, i} \times q_{\mathrm{food}, i}}{B_{\mathrm{W}}}
$$

Where $C_{\text {food, } i}$ is the average concentration of the respective PFAAs in each food category ( $\mathrm{ng} \mathrm{g}^{-1}$ wet weight), $q_{\text {food }, i}$ is the estimated quantity of food consumed per day of a specific food 

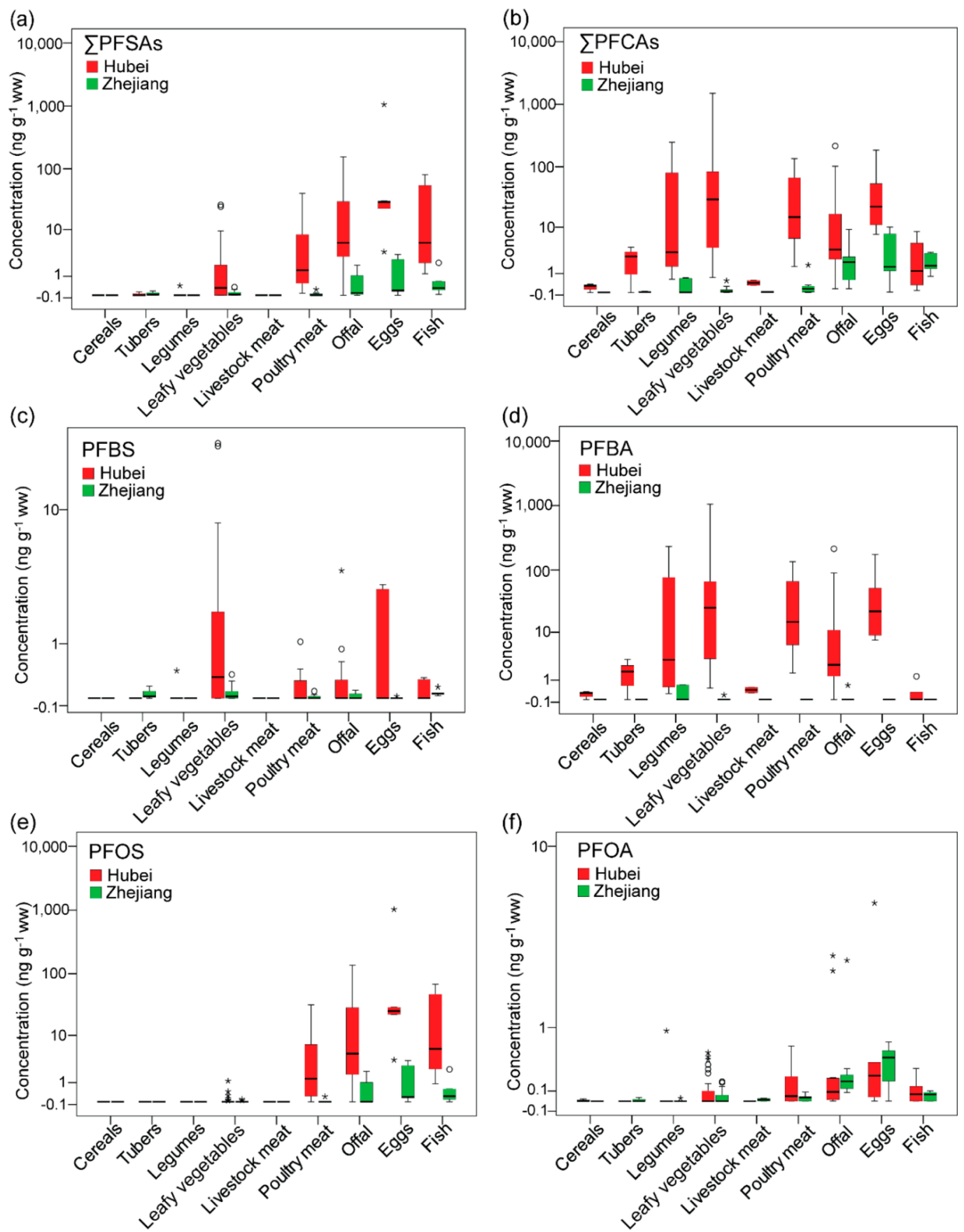

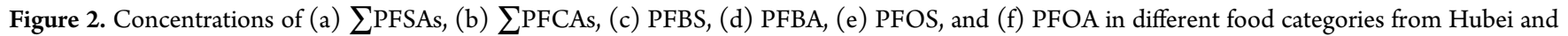
Zhejiang province, respectively. It should be noted that the scales of the $y$-axis vary for the different PFAAs due to the large variability in concentrations.

category $\left(\mathrm{g} \mathrm{day}^{-1}\right)$ and $B_{\mathrm{w}}$ is the body weight $(\mathrm{kg})$. The average daily intake of each food category for male adults in the two investigated regions and adults at different ages ${ }^{29}$ are shown in SI Table S6 and Table S7, respectively. For censored concentration data, we applied a lower bound (LB) and upper bound (UB) approach where nondetects were assigned as zero or half the MDL respectively.

2.7. Statistical Analysis. Statistical analysis was performed using PASW V18.0 (SPSS Inc.) and Excel (Microsoft Inc.). Differences in concentrations of PFAAs in the food categories from the different regions were evaluated using the nonparametric Mann-Whitney U-test. Correlation analysis was performed with Spearman's rank correlation coefficient $(\rho)$. Tests showing significance levels $<0.05$ were considered as statistically significant.

\section{RESULTS AND DISCUSSION}

3.1. Concentrations in Food Items. Detection frequencies (DFs) of PFAAs varied greatly among the different food categories and sampling locations (see SI Table S8). Overall, long-chain PFCAs including PFOA, PFNA, PFUnDA, PFDoDA, and PFTrDA were detected in eggs, fish, fish liver, and other offals at comparable frequencies between the two provinces (DF $\geq 50 \%$ ) whereas short-chain PFCAs and PFBS 
were primarily detected in leafy vegetables, legumes, and tubers from Hubei province (DF > 67\%). PFOS displayed a high detection frequency (DF $\geq 78 \%$ ) in eggs, fish and fish liver from both sampling locations while PFHxS was most frequently detected in animal food samples from Hubei province. PFDcS and PFOSA were below detection limits in the majority of samples $(\mathrm{DF}<3 \%)$ and thus were not included in the following discussion.

Total concentrations of $\sum$ PFAAs from both sampling locations displayed large variability, but were considerably higher in all food categories from Hubei (range, average, median: < MDL-1523, 97.3, $\left.12.8 \mathrm{ng} \mathrm{g}^{-1} \mathrm{ww}\right)$, compared to Zhejiang (<MDL-14.3, 1.71, $0.17 \mathrm{ng} \mathrm{g}^{-1} \mathrm{ww}$ ) (Figure 2 and SI Figure S1). Concentrations of $\sum$ PFSAs were generally higher in foods of animal origin while the highest concentrations of $\sum$ PFCAs were detected in foods of plant origin (Figure 2). The findings of PFOS and long-chain PFCAs as the predominant compounds in fish samples are well in line with the positive relationship between perfluoroalkyl chainlength and bioaccumulation potential in the aquatic environment. ${ }^{6,37}$ A similar PFAA profile in other animal food products including eggs, offal and poultry meat provide support to recent studies showing that PFOS and some long-chain PFCAs also bioaccumulate in terrestrial agricultural food chains. ${ }^{38-40}$ The higher levels in eggs and offal food compared to meat are further consistent with preferential distribution to the liver and eggs compared to muscle tissue. ${ }^{41,42}$ The distinct PFAA profile in leafy vegetables (PFBA $>$ PFPA $>$ PFBS $>$ PFHxA $>$ PFHpA $\gg$ long-chain PFAAs) compared to animal food items indicate that other mechanisms are responsible for the accumulation of PFAAs in edible parts of plants leading to subsequent human exposure (Figure 2 and SI Figure S1). In contrast to food items from animal products, where increasing hydrophobicity and proteinophilicity leads to slow elimination, ${ }^{6,43}$ the high water solubility of short-chain homologues facilitates efficient uptake from pore water and translocation within the plant. ${ }^{44-48}$ As the water evaporates, the anionic and nonvolatile PFAAs will subsequently be enriched in the plant material. ${ }^{44-48}$ Interestingly, the levels of PFAAs in leafy vegetables (Chinese cabbage, leek, spinach, greens, Chinese kale) were higher than those in tubers (white radish, carrot, sweet potato) and fruit vegetables (tomato, pumpkin, hot pepper) with exception of hyacinth bean. Thus, the measured concentrations of short-chain PFAAs are generally consistent with controlled uptake experiments showing that the evapotranspiration at the leaves lead to the highest accumulation factors in plants. ${ }^{46,49-52}$

Geographical differences in PFAA concentrations between two regions or sampling sites within one region were highly homologue-specific and varied among the food groups (Figure $2 \mathrm{c}-\mathrm{f}$ and SI Figure $\mathrm{S} 1 \mathrm{~b}-\mathrm{k})$. The most pronounced geographical differences were observed for PFBS, PFHxS, PFOS, PFBA, PFPA and PFHxA which were typically $1-2$ orders of magnitude higher in samples from Hubei province compared to Zhejiang province. The elevated PFOS, PFHxS, and PFBS concentrations in food samples from Hubei province compared to Zhejiang province were somewhat expected, since these substances are currently produced in the area. ${ }^{15}$ The elevated concentrations of short-chain PFCAs are also in agreement with previous measurement in river water from this area. ${ }^{53}$ In contrast to PFSAs and short-chain PFCAs, the concentrations of C8-C13 PFCAs were comparable between the two provinces (median concentrations in different food categories within a factor of 2) and some food categories even showed higher levels for Zhejiang compared to Hubei. This may be explained by fluoropolymer manufacturing facilities located upstream the Qiantang river or industrial use of telomer-based precursors which can be degraded to form long-chain PFCAs. SI Figure S2a-d displays the spatial trends for the different sampling sites in Hubei and Zhejiang, respectively. Strong correlations between a large number of PFAAs (SI Table S9) and decreasing concentrations with increasing distance to the PFAAs production facility indicate that the production facility was an important point source also for short-chain PFCAs (SI Figure S2a-b). The concurrent emissions of PFCAs from this facility, which primarily produces PFSAs, may be attributed to impurities and/or degradation products from the manufacturing process. $5,27,53$ However, it is also possible that the production inventory for this particular plant is incomplete and manufacture of additional fluorochemical products may help to explain the high levels of short-chain PFCAs. The lack of a clear spatial trend of PFSAs and PFCAs among sampling sites in Zhejiang (SI Figure $\mathrm{S} 2 \mathrm{c}-\mathrm{d}$ ) indicate that there are no distinct point source within the sampling area.

3.2. Total Dietary Intake of PFAAs. Based on the PFAA concentrations in different food categories and site-specific intake data, EDIs were estimated for the adult population in Hubei and Zhejiang province, respectively. As shown in Table 1, the total dietary intake of $\sum$ PFAAs in Hubei province (998

Table 1. Average Estimated Daily Intake (EDI) of PFAA Compounds from Foods for Adults in Hubei and Zhejiang $\left(\mathrm{ng} \mathrm{kg}^{-1} \mathrm{day}^{-1}\right)^{a, b, c, d}$

\begin{tabular}{|c|c|c|c|c|}
\hline \multirow[b]{2}{*}{ compounds } & \multicolumn{2}{|c|}{$\begin{array}{l}\text { EDI for point source area } \\
\text { in Hubei }\end{array}$} & \multicolumn{2}{|c|}{$\begin{array}{l}\text { EDI for application area } \\
\text { in Zhejiang }\end{array}$} \\
\hline & $\begin{array}{c}\text { lower } \\
\text { bound } \mathrm{N} \\
D=0\end{array}$ & $\begin{array}{l}\text { upper bound } \mathrm{N} \\
D=1 / 2 \mathrm{MDL}\end{array}$ & $\begin{array}{c}\text { lower } \\
\text { bound } \mathrm{N} \\
D=0\end{array}$ & $\begin{array}{l}\text { upper bound N } \\
D=1 / 2 \mathrm{MDL}\end{array}$ \\
\hline PFBS & 12.2 & 13.4 & 0.39 & 0.46 \\
\hline PFHxS & 5.29 & 5.36 & 0.01 & 0.11 \\
\hline PFOS & 86.7 & 87.5 & 1.66 & 3.25 \\
\hline PFBA & 682.2 & 682.5 & 0.21 & 1.40 \\
\hline PFPA & 128.3 & 128.3 & 0.08 & 0.14 \\
\hline PFHxA & 76.3 & 76.4 & 0.01 & 0.23 \\
\hline PFHpA & 2.94 & 2.98 & 0.003 & 0.08 \\
\hline PFOA & 1.15 & 1.18 & 0.59 & 0.71 \\
\hline PFNA & 0.45 & 0.48 & 0.34 & 0.39 \\
\hline PFDcA & 0.74 & 0.82 & 1.83 & 1.94 \\
\hline PFUnDA & 1.05 & 1.08 & 1.47 & 1.53 \\
\hline PFDoDA & 0.13 & 0.17 & 0.78 & 0.84 \\
\hline PFTrDA & 0.24 & 0.27 & 1.05 & 1.10 \\
\hline PFTeDA & 0.01 & 0.18 & 0.58 & 0.81 \\
\hline$\sum$ PFAAs & 997.9 & 1001 & 9.03 & 14.5 \\
\hline
\end{tabular}

${ }^{a}$ EDI calculated for male adults with an assumed average body weight $61 \mathrm{~kg} .{ }^{b}$ Calculations were not performed for PFDcS and PFOSA due to the low detection frequencies $(<3 \%)$. ${ }^{c}$ Fish liver were not included into the calculation of EDI due to lack of consumption data. ${ }^{d}$ The lower bounds (LB) and upper bounds (UB) of average EDIs were calculated by using zero or half the MDL to substitute values of nondetects, respectively.

$\mathrm{ng} \mathrm{kg}{ }^{-1}$ day $^{-1}$ ) was more than 2 orders of magnitude higher than in Zhejiang province $\left(9.03 \mathrm{ng} \mathrm{kg}^{-1} \mathrm{day}^{-1}\right)$ in the lower bound scenario. In Hubei province, the largest contribution to the EDI for $\sum$ PFAAs was from PFBA, PFPA, PFHxA, and PFOS whereas EDI for $\sum$ PFAAs in Zhejiang province was dominated by PFDcA, PFUnDA, PFTrDA and PFOS. The 
percentage difference in $\Sigma$ PFAAs dietary intakes between the upper- and lower-bound scenario for Hubei province was $0.3 \%$ demonstrating that nondetects had a negligible influence on the EDI calculations. A larger difference between the upper- and lower-bound scenario (37.8\%) was observed for Zhejiang province indicating that improvements in analytical techniques and detection frequency could reduce the uncertainty in calculated EDIs.

In Figure 3, the relative contribution of different food categories to total dietary intakes are presented. Leafy

(a) Hubei

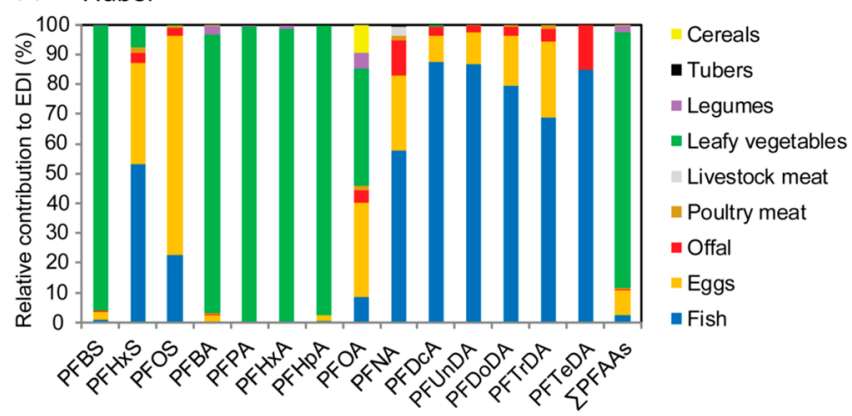

(b) Zhejiang

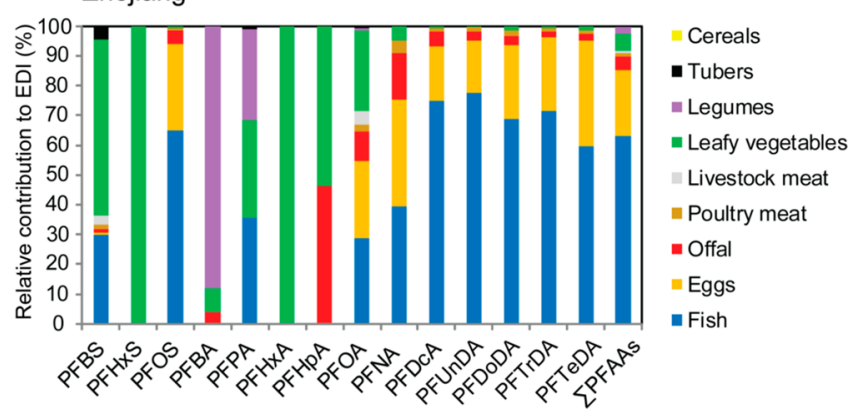

Figure 3. Relative contribution of different food categories to EDIs for different PFAA homologues in samples from (a) Hubei and (b) Zhejiang, respectively.

vegetables were the main source of $\sum$ PFAAs in Hubei province while fish and eggs dominated the dietary intake of $\sum$ PFAAs in Zhejiang province. These differences were primarily due to the geographical difference in PFAA concentrations (as discussed above), but they also reflect differences in dietary habits between the provinces. For instance, the population in Hubei had a significantly lower consumption of fish and meat and higher consumption of leafy vegetables compared to Zhejiang (SI Table S6). Despite these differences in consumption habits, the relative importance of different food categories was relatively consistent between the two provinces when considering individual PFAAs. Fish, eggs, and offal were the dominating sources of dietary intake for PFOS and C9-C13 PFCAs whereas leafy vegetables or legumes dominated the intake of PFBS and short-chain PFCAs. This demonstrates that while the magnitude of exposure to PFAAs is strongly influenced by local emission sources (as shown in Table 1), the dietary exposure pathways of different PFAAs are primarily governed by the intrinsic chemical properties leading to accumulation in plants and animals respectively (as discussed above). For PFOA and PFHxS there were, however, significant contributions to the total dietary intake from both animal food products (eggs, fish, and offal) and plants (leafy vegetables, legumes, and cereals) and some differences in the dominating dietary exposure pathway between the two provinces. This can be explained by the fact that these compounds are moderately water-soluble and not strongly bioaccumulative in aquatic food chains. ${ }^{6}$

3.3. Comparison with Dietary Intake Assessments from Other Countries. In order to put the EDIs calculated here into a global perspective, a comparison with previous assessments from Europe, ${ }^{22,54-57}$ North America, ${ }^{58-60}$ Japan, ${ }^{61}$ and Korea $^{62}$ is provided in SI Table S10. Overall, EDIs of $\sum$ PFAAs reported in all previous studies vary between $0.64-$ $22.0 \mathrm{ng} \mathrm{kg}^{-1} \mathrm{day}^{-1}$. In this context, the EDIs for Hubei province represent highly elevated exposures exceeding previous studies by several orders of magnitude. The high EDIs in Hubei are consistent with biomonitoring studies reporting the highest serum concentrations of PFOS ever measured in nonoccupationally exposed humans from a population of high fish consumers around Tangxun Lake. ${ }^{10}$ Collectively, these two studies, which were conducted in different parts of Hubei province, demonstrate that the emissions from ongoing PFAAsproduction in China lead to some of the highest PFAA exposures in the world. Considering that there are currently about 15 enterprises in China producing POSF (the majority in Hubei and Fujian province) $)^{15}$ studies to assess exposure in these hot spot areas including additional pathways such as drinking water and dust are strongly encouraged. Two previous studies on PFAAs surrounding the same point source in Hubei have shown that PFAAs in tap water (as drinking water) was not detectable or at very low levels, indicating that PFAAs intake from solid foods may play a more important role of dietary exposure in this region. ${ }^{27,63}$ However, when drinking water is produced by using groundwater, point sources might play a role in the overall exposure. ${ }^{64}$ The source of drinking water in relation to the point source is often unclear, making any assumptions challenging. A more detailed approach covering the dietary contribution of drinking water to the Chinese population is needed but was beyond the scope of this study. Other routes of exposure as for example exposure through skin contact and inhalation, due to contact with consumer products, may also be significant contributors to overall exposure.

The EDIs of $\sum$ PFAAs ( $\mathrm{LB}=9.03 \mathrm{ng} \mathrm{kg}^{-1} \mathrm{day}^{-1}$; UB $=14.5$ $\mathrm{ng} \mathrm{kg}^{-1} \mathrm{day}^{-1}$ ) from Zhejiang province were also in the higher range of previous studies. When comparing EDIs from different studies it is, however, important to consider the influence of analytical protocols and treatment of nondetects in the data sets. For example, it is likely that EDIs reported in recent studies are more robust as the development of analytical techniques has improved detection frequency, precision, and accuracy of PFAAs in food. ${ }^{34,65}$ EDIs may also be affected by the sample collection method and calendar year that the study was performed. The standard approach to estimate dietary intake is by collection and analysis of composite foods (sometimes referred to as food basket samples) where a large number of commonly consumed food items are pooled. ${ }^{56-60}$ Many studies, including this one, collected and analyzed individual food items which are subsequently aggregated into food categories for calculation of EDIs. ${ }^{22,55,62}$ A third approach, which is used more seldom, is the duplicate diet method where duplicate food portions consumed by one individual during 1 day are pooled and analyzed as a homogenate. ${ }^{54,61}$ When comparing our results with Klenow et al., ${ }^{55}$ who applied similar food sampling strategy, analytical approach and EDI calculations as in this study, the lower bound EDIs in Zhejiang 
province appear to be approximately an order of magnitude higher than those in Europe ( $\mathrm{LB}=0.58 \mathrm{ng} \mathrm{kg}^{-1} \mathrm{day}^{-1}$; UB = $\left.1.14 \mathrm{ng} \mathrm{kg}^{-1} \mathrm{day}^{-1}\right)$. This may be an indication of an overall higher EDIs of PFAAs in China compared to other countries. However, due to the methodological aspects mentioned above and large variability between different studies the comparison of EDIs should be interpreted with caution.

3.4. Implications for Human Health Risks of PFAA Exposure. The high EDIs in Hubei province also warrants an assessment of the human health risks associated with this exposure. Although, no guidelines for dietary intake of PFAAs exist in China, health-based intake values for PFOS have been established in other parts of the world. The European Food Safety Agency (EFSA) has set the tolerable daily intake (TDI) of PFOS to $150 \mathrm{ng} \mathrm{kg}^{-1} \mathrm{day}^{-1}$ based on the no observed adverse effect level from a subchronic study in cynomolgous monkeys. ${ }^{66}$ TDIs of PFOS suggested by the UK Committee on Toxicity of Chemicals in Food and German Federal Institute for Risk Assessment are $300 \mathrm{ng} \mathrm{kg}^{-1}$ day $^{-1}$ and $100 \mathrm{ng} \mathrm{kg}^{-1}$ day $^{-1}$, respectively. ${ }^{67,68}$ A more recent health advisory from the United States Environmental Protection Agency used substantially lower reference doses $\left(20 \mathrm{ng} \mathrm{kg}^{-1}\right.$ day $\left.^{-1}\right)$ for both PFOA and PFOS derived from developmental effects in rats. ${ }^{69}$ Based on these health advisory assessments it can be concluded that the EDIs for PFOS in Hubei province $\left(87 \mathrm{ng} \mathrm{kg}^{-1}\right.$ day ${ }^{-1}$ ) are associated with risks for adverse human health effects. A comprehensive analysis on EDIs determined for different age classes in Hubei (SI Table S11), also show that the EDIs of children (2-14 years of age) are up to two times higher than adults which makes this group particularly susceptible to effects from long-term dietary exposure to PFAAs.

Another important aspect when evaluating human health implications of PFAA exposure for the Hubei population is that there are currently no established TDIs for short-chain PFCAs and PFBS. As these substances contributed to more than $90 \%$ of the $\sum$ PFAA EDIs in Hubei province the comparison with advisory guideline for PFOS alone may greatly underestimate the human health risks. In a cumulative risk assessment framework for 17 per- and polyfluoroalkyl substances developed by Borg et al. ${ }^{70}$ it was assumed that the toxicity of short-chain PFAAs can be extrapolated from long-chain homologues based on internal dose (i.e., serum concentrations). By applying this assumption to the exposure situation in Hubei province it seems likely that the high EDIs of short-chain PFAAs would contribute to the cumulative health risks of PFAAs despite a more rapid elimination than PFOS. ${ }^{10}$ So far, the low bioaccumulation potential in fish and more rapid urinary clearance of short-chain PFAAs in humans has been the main reasons for the fluorochemical industry to promote this group of chemicals as safe substitutes. ${ }^{12}$ This study, however, demonstrates that accumulation in plants and subsequent dietary exposure are important mechanisms that need to be considered for accurate risk assessment of short-chain PFAAs.

\section{ASSOCIATED CONTENT}

\section{S Supporting Information}

The Supporting Information is available free of charge on the ACS Publications website at DOI: 10.1021/acs.est.7b00246.

Additional information as noted in the text (PDF)

\section{AUTHOR INFORMATION}

\section{Corresponding Authors}

*(T.W.) Phone: +46 19303 462; e-mail: thanh.wang@oru.se.

*(D.H.) Phone: +47 47267 182; e-mail: dorte.herzke@nilu.no. ORCID $\odot$

Thanh Wang: 0000-0002-5729-1908

Dorte Herzke: 0000-0001-9620-3053

\section{Notes}

The authors declare no competing financial interest.

\section{ACKNOWLEDGMENTS}

We gratefully acknowledge Dr. Sandra Huber from NILU for assisting in the PFAA measurements. We also thank External Cooperation Program of the Chinese Academy of Sciences (GJHZ1202) and the Norwegian Research Council for funding (209666/E40). Additionally, the National Natural Science Foundation of China (21507113) and the Natural Science Foundation of Zhejiang Province (LY15B070006) are acknowledged for their financial support.

\section{REFERENCES}

(1) Buck, R. C.; Franklin, J.; Berger, U.; Conder, J. M.; Cousins, I. T.; de Voogt, P.; Jensen, A. A.; Kannan, K.; Mabury, S. A.; van Leeuwen, S. P. Perfluoroalkyl and polyfluoroalkyl substances in the environment: terminology, classification, and origins. Integr. Environ. Assess. Manage. 2011, 7 (4), 513-541.

(2) Lindstrom, A. B.; Strynar, M. J.; Libelo, E. L.; Field, J. A. Guest comment: Perfluoroalkyl acid focus issue. Environ. Sci. Technol. 2011, 45 (19), 7951-7953.

(3) Synthesis Paper on Per-And Polyfluorinated Chemicals (PFCs); OECD/UNEP Global PFC Group, Environment Health and Safety, Environment Directorate, OECD: Paris, France, 2013; www.oecd.org/ env/ehs/risk-management/PFC_FINAL-Web.pdf.

(4) US EPA 2010/2015 PFOA Stewardship Program. Available from: https://www.epa.gov/assessing-and-managing-chemicals-under-tsca/ and-polyfluoroalkyl-substances-pfass-under-tsca\#tab-3. 2006.

(5) Wang, Z.; Cousins, I. T.; Scheringer, M.; Hungerbühler, K. Fluorinated alternatives to long-chain perfluoroalkyl carboxylic acids (PFCAs), perfluoroalkane sulfonic acids (PFSAs) and their potential precursors. Environ. Int. 2013, 60 (5), 242-248.

(6) Conder, J. M.; Hoke, R. A.; de Wolf, W.; Russell, M. H.; Buck, R. C. Are PFCAs bioaccumulative? A critical review and comparison with regulatory criteria and persistent lipophilic compounds. Environ. Sci. Technol. 2008, 42 (4), 995-1003.

(7) Russell, M. H.; Nilsson, H.; Buck, R. C. Elimination kinetics of perfluorohexanoic acid in humans and comparison with mouse, rat and monkey. Chemosphere 2013, 93 (10), 2419-2425.

(8) Chang, S.-C.; Das, K.; Ehresman, D. J.; Ellefson, M. E.; Gorman, G. S.; Hart, J. A.; Noker, P. E.; Tan, Y.-M.; Lieder, P. H.; Lau, C.; Olsen, G. W.; Butenhoff, J. L. Comparative pharmacokinetics of perfluorobutyrate in rats, mice, monkeys, and humans and relevance to human exposure via drinking water. Toxicol. Sci. 2008, 104 (1), 40-53.

(9) Olsen, G. W.; Chang, S.-C.; Noker, P. E.; Gorman, G. S.; Ehresman, D. J.; Lieder, P. H.; Butenhoff, J. L. A comparison of the pharmacokinetics of perfluorobutanesulfonate (PFBS) in rats, monkeys, and humans. Toxicology 2009, 256 (1-2), 65-74.

(10) Zhou, Z.; Shi, Y.; Vestergren, R.; Wang, T.; Liang, Y.; Cai, Y. Highly elevated serum concentrations of perfluoroalkyl substances in fishery employees from Tangxun Lake, China. Environ. Sci. Technol. 2014, 48 (7), 3864-3874.

(11) Blum, A.; Balan, S. A.; Scheringer, M.; Trier, X.; Goldenman, G.; Cousins, I. T.; Diamond, M.; Fletcher, T.; Higgins, C.; Lindeman, A. E.; Peaslee, G.; de Voogt, P.; Wang, Z.; Weber, R. The Madrid statement on poly- and perfluoroalkyl substances (PFASs). Environ. Health Perspect. 2015, 123 (5), A107-A111. 
(12) Bowman, J. S. Response to "Comment on 'Fluorotechnology is critical to modern life: The FluoroCouncil Counterpoint to the Madrid statement". Environ. Health Perspect. 2015, 123 (7), A170A171.

(13) Shi, Y.; Vestergren, R.; Zhou, Z.; Song, X.; Xu, L.; Liang, Y.; Cai, $\mathrm{Y}$. Tissue distribution and whole body burden of the chlorinated polyfluoroalkyl ether sulfonic acid F-53B in crucian carp (Carassius carassius): Evidence for a highly bioaccumulative contaminant of emerging concern. Environ. Sci. Technol. 2015, 49 (24), 14156-14165. (14) Shi, Y.; Vestergren, R.; Xu, L.; Zhou, Z.; Li, C.; Liang, Y.; Cai, Y. Human exposure and elimination kinetics of chlorinated polyfluoroalkyl ether sulfonic acids (Cl-PFESAs). Environ. Sci. Technol. 2016, 50 (5), 2396-2404.

(15) Xie, S.; Wang, T.; Liu, S.; Jones, K. C.; Sweetman, A. J.; Lu, Y. Industrial source identification and emission estimation of perfluorooctane sulfonate in China. Environ. Int. 2013, 52, 1-8.

(16) Li, L.; Zhai, Z.; Liu, J.; Hu, J. Estimating industrial and domestic environmental releases of perfluorooctanoic acid and its salts in China from 2004 to 2012. Chemosphere 2015, 129, 100-109.

(17) Heydebreck, F.; Tang, J.; Xie, Z.; Ebinghaus, R. Alternative and legacy perfluoroalkyl substances: Differences between European and Chinese river/estuary systems. Environ. Sci. Technol. 2015, 49 (14), 8386-8395.

(18) Shi, Y.; Vestergren, R.; Xu, L.; Song, X.; Niu, X.; Zhang, C.; Cai, Y. Characterizing direct emissions of perfluoroalkyl substances from ongoing fluoropolymer production sources: A spatial trend study of Xiaoqing River, China. Environ. Pollut. 2015, 206, 104-112.

(19) Wang, P.; Lu, Y.; Wang, T.; Meng, J.; Li, Q.; Zhu, Z.; Sun, Y.; Wang, R.; Giesy, J. P. Shifts in production of perfluoroalkyl acids affect emissions and concentrations in the environment of the Xiaoqing River Basin, China. J. Hazard. Mater. 2016, 307, 55-63.

(20) Trudel, D.; Horowitz, L.; Wormuth, M.; Scheringer, M.; Cousins, I. T.; Hungerbuhler, K. Estimating consumer exposure to PFOS and PFOA. Risk Anal. 2008, 28 (2), 251-269.

(21) Egeghy, P. P.; Lorber, M. An assessment of the exposure of Americans to perfluorooctane sulfonate: A comparison of estimated intake with values inferred from NHANES data. J. Exposure Sci. Environ. Epidemiol. 2011, 21, 150-168.

(22) Haug, L. S.; Thomsen, C.; Brantsæeter, A. L.; Kvalem, H. E.; Haugen, M.; Becher, G.; Alexander, J.; Meltzer, H. M.; Knutsen, H. K. Diet and particularly seafood are major sources of perfluorinated compounds in humans. Environ. Int. 2010, 36 (7), 772-778.

(23) Wu, Y.; Wang, Y.; Li, J.; Zhao, Y.; Guo, F.; Liu, J.; Cai, Z. Perfluorinated compounds in seafood from coastal areas in China. Environ. Int. 2012, 42, 67-71.

(24) Zhang, T.; Sun, H. W.; Wu, Q.; Zhang, X. Z.; Yun, S. H.; Kannan, K. Perfluorochemicals in meat, eggs and indoor dust in China: Assessment of sources and pathways of human exposure to perfluorochemicals. Environ. Sci. Technol. 2010, 44 (9), 3572-3579.

(25) Wang, J. M.; Shi, Y. L.; Pan, Y. Y.; Cai, Y. Q. Perfluorinated compounds in milk, milk powder and yoghurt purchased from markets in China. Chin. Sci. Bull. 2010, 55 (11), 1020-1025.

(26) Shan, G.; Wang, Z.; Zhou, L.; Du, P.; Luo, X.; Wu, Q.; Zhu, L. Impacts of daily intakes on the isomeric profiles of perfluoroalkyl substances (PFASs) in human serum. Environ. Int. 2016, 89-90, 6270 .

(27) Gao, Y.; Fu, J.; Cao, H.; Wang, Y.; Zhang, A.; Liang, Y.; Wang, T.; Zhao, C.; Jiang, G. Differential accumulation and elimination behavior of perfluoroalkyl acid isomers in occupational workers in a manufactory in China. Environ. Sci. Technol. 2015, 49 (11), 69536962.

(28) Zhai, F.; Wang, H.; Du, S.; He, Y.; Wang, Z.; Ge, K.; Popkin, B. M. Lifespan nutrition and changing socio-economic conditions in China. Asia Pac. J. Clin. Nutr. 2007, 16 (Suppl 1), 374-382.

(29) Jin, S. G., Ed. In Part 10th of Survey of Nutrition and Health Status of Chinese Residents: Data for Nutrition and Health, 2002; People's Medical Publishing House: Beijing, 2008.

(30) Wang, T.; Vestergren, R.; Herzke, D.; Yu, J.; Cousins, I. T. Levels, isomer profiles, and estimated riverin mass discharges of perfluoroalkyl acids and fluorinated alternatives at the mouths of Chinese rivers. Environ. Sci. Technol. 2016, 50 (21), 11584-11592.

(31) Wang, T.; Wang, P.; Meng, J.; Liu, S.; Lu, Y.; Khim, J. S.; Giesy, J. P. A review of sources, multimedia distribution and health risks of perfluoroalkyl acids (PFAAs) in China. Chemosphere 2015, 129, 8799.

(32) Vestergren, R.; Ullah, S.; Cousins, I. T.; Berger, U. A matrix effect-free method for reliable quantification of perfluoroalkyl carboxylic acids and perfluoroalkane sulfonic acids at low parts per trillion levels in dietary samples. J. Chromatogr. A 2012, 1237, 64-71.

(33) Hanssen, L.; Dudarev, A. A.; Huber, S.; Odland, J. Ø.; Nieboer, E.; Sandanger, T. M. Partition of perfluoroalkyl substances (PFASs) in whole blood and plasma, assessed in maternal and umbilical cord samples from inhabitants of arctic Russia and Uzbekistan. Sci. Total Environ. 2013, 447, 430-437.

(34) Weiss, J. M.; van der Veen, I.; de Boer, J.; van Leeuwen, S. P. J.; Cofino, W.; Crum, S. Analytical improvements shown over four interlaboratory studies of perfluoroalkyl substances in environmental and food samples. TrAC, Trends Anal. Chem. 2013, 43, 204-216.

(35) Herzke, D.; Huber, S.; Bervoets, L.; D’Hollander, W.; Hajslova, J.; Pulkrabova, J.; Brambilla, G.; De Filippis, S. P.; Klenow, S.; Heinemeyer, G.; de Voogt, P. Perfluorinated alkylated substances in vegetables collected in four European countries; occurrence and human exposure estimations. Environ. Sci. Pollut. Res. 2013, 20 (11), 7930-7939.

(36) Ullah, S.; Alsberg, T.; Vestergren, R.; Berger, U. Determination of perfluoroalkyl carboxylic, sulfonic, and phosphonic acids in food. Anal. Bioanal. Chem. 2012, 404 (8), 2193-2201.

(37) Martin, J. W.; Mabury, S. A.; Solomon, K. R.; Muir, D. C. G. Dietary accumulation of perfluorinated acids in juvenile rainbow trout (Oncorhynchus mykiss). Environ. Toxicol. Chem. 2003, 22 (1), 189-195.

(38) Vestergren, R.; Orata, F.; Berger, U.; Cousins, I. T. Bioaccumulation of perfluoroalkyl acids in dairy cows in a naturally contaminated environment. Environ. Sci. Pollut. Res. 2013, 20 (11), 7959-7969.

(39) Kowalczyk, J.; Ehlers, S.; Oberhausen, A.; Tischer, M.; Fuerst, P.; Schafft, H.; Lahrssen-Wiederholt, M. Absorption, distribution, and milk secretion of the perfluoroalkyl acids PFBS, PFHxS, PFOS, and PFOA by dairy cows fed naturally contaminated feed. J. Agric. Food Chem. 2013, 61 (12), 2903-2912.

(40) Numata, J.; Kowalczyk, J.; Adolphs, J.; Ehlers, S.; Schafft, H.; Fuerst, P.; Müeller-Graf, C.; Lahrssen-Wiederholt, M.; Greiner, M. Toxicokinetics of seven perfluoroalkyl sulfonic and carboxylic acids in pigs fed a contaminated diet. J. Agric. Food Chem. 2014, 62 (28), 6861-6870.

(41) Yoo, H.; Guruge, K. S.; Yamanaka, N.; Sato, C.; Mikami, O.; Miyazaki, S.; Yamashita, N.; Giesy, J. P. Depuration kinetics and tissue disposition of PFOA and PFOS in white leghorn chickens (Gallus gallus) administered by subcutaneous implantation. Ecotoxicol. Environ. Saf. 2009, 72 (1), 26-36.

(42) Holmstrom, K. E.; Berger, U. Tissue distribution of perfluorinated surfactants in common guillemot (Uria aalge) from the Baltic Sea. Environ. Sci. Technol. 2008, 42 (16), 5879-5884.

(43) $\mathrm{Ng}$, C. A.; Hungerbühler, K. Bioaccumulation of perfluorinated alkyl acids: Observations and models. Environ. Sci. Technol. 2014, 48 (9), 4637-4648.

(44) Felizeter, S.; McLachlan, M. S.; de Voogt, P. Uptake of perfluorinated alkyl acids by hydroponically grown lettuce (Lactuca sativa). Environ. Sci. Technol. 2012, 46 (21), 11735-11743.

(45) Blaine, A. C.; Rich, C. D.; Sedlacko, E. M.; Hyland, K. C.; Stushnoff, C.; Dickenson, E. R. V.; Higgins, C. P. Perfluoroalkyl acid uptake in lettuce (Lactuca sativa) and strawberry (Fragaria ananassa) irrigated with reclaimed water. Environ. Sci. Technol. 2014, 48 (24), 14361-14368.

(46) Felizeter, S.; McLachlan, M. S.; De Voogt, P. Root uptake and translocation of perfluorinated alkyl acids by three hydroponically grown crops. J. Agric. Food Chem. 2014, 62 (15), 3334-3342.

(47) Mueller, C. E.; LeFevre, G. H.; Timofte, A. E.; Hussain, F. A.; Sattely, E. S.; Luthy, R. G. Competing mechanisms for perfluoroalkyl 
acid accumulation in plants revealed using an Arabidopsis model system. Environ. Toxicol. Chem. 2016, 35 (5), 1138-1147.

(48) Krippner, J.; Brunn, H.; Falk, S.; Georgii, S.; Schubert, S.; Stahl, T. Effects of chain length and $\mathrm{pH}$ on the uptake and distribution of perfluoroalkyl substances in maize (Zea mays). Chemosphere 2014, 94, $85-90$.

(49) Zhao, S.; Fang, S.; Zhu, L.; Liu, L.; Liu, Z.; Zhang, Y. Mutual impacts of wheat (Triticum aestivum L.) and earthworms (Eisenia fetida) on the bioavailability of perfluoroalkyl substances (PFASs) in soil. Environ. Pollut. 2014, 184, 495-501.

(50) Blaine, A. C.; Rich, C. D.; Hundal, L. S.; Lau, C.; Mills, M. A.; Harris, K. M.; Higgins, C. P. Uptake of perfluoroalkyl acids into edible crops via land applied biosolids: Field and greenhouse studies. Environ. Sci. Technol. 2013, 47 (24), 14062-14069.

(51) Wen, B.; Li, L.; Zhang, H.; Ma, Y.; Shan, X.-Q.; Zhang, S. Field study on the uptake and translocation of perfluoroalkyl acids (PFAAs) by wheat (Triticum aestivum L.) grown in biosolids-amended soils. Environ. Pollut. 2014, 184, 547-554.

(52) García-Valcárcel, A. I.; Molero, E.; Escorial, M. C.; Chueca, M. C.; Tadeo, J. L. Uptake of perfluorinated compounds by plants grown in nutrient solution. Sci. Total Environ. 2014, 472, 20-26.

(53) Wang, Y.; Fu, J.; Wang, T.; Liang, Y.; Pan, Y.; Cai, Y.; Jiang, G. Distribution of perfluorooctane sulfonate and other perfluorochemicals in the ambient environment around a manufacturing facility in China. Environ. Sci. Technol. 2010, 44 (21), 8062-8067.

(54) Fromme, H.; Schlummer, M.; Möller, A.; Gruber, L.; Wolz, G.; Ungewiss, J.; Böhmer, S.; Dekant, W.; Mayer, R.; Liebl, B.; Twardella, D. Exposure of an adult population to perfluorinated substances using duplicate diet portions and biomonitoring data. Environ. Sci. Technol. 2007, 41 (22), 7928-7933.

(55) Klenow, S.; Heinemeyer, G.; Brambilla, G.; Dellatte, E.; Herzke, D.; de Voogt, P. Dietary exposure to selected perfluoroalkyl acids (PFAAs) in four European regions. Food Addit. Contam., Part A 2013, 30 (12), 2141-2151.

(56) Noorlander, C. W.; van Leeuwen, S. P. J.; te Biesebeek, J. D.; Mengelers, M. J. B.; Zeilmaker, M. J. Levels of perfluorinated compounds in food and dietary intake of PFOS and PFOA in the Netherlands. J. Agric. Food Chem. 2011, 59 (13), 7496-7505.

(57) Gebbink, W. A.; Glynn, A.; Darnerud, P. O.; Berger, U. Perfluoroalkyl acids and their precursors in Swedish food: The relative importance of direct and indirect dietary exposure. Environ. Pollut. 2015, 198, 108-115.

(58) Ostertag, S. K.; Tague, B. A.; Humphries, M. M.; Tittlemier, S. A.; Chan, H. M. Estimated dietary exposure to fluorinated compounds from traditional foods among Inuit in Nunavut, Canada. Chemosphere 2009, 75 (9), 1165-1172.

(59) Schecter, A.; Colacino, J.; Haffner, D.; Patel, K.; Opel, M.; Päpke, O.; Birnbaum, L. Perfluorinated compounds, polychlorinated biphenyls, and organochlorine pesticide contamination in composite food samples from Dallas, Texas, USA. Environ. Health Perspect. 2010, 118 (6), 796-802.

(60) Tittlemier, S. A.; Pepper, K.; Seymour, C.; Moisey, J.; Bronson, R; Cao, X.-L.; Dabeka, R. W. Dietary exposure of Canadians to perfluorinated carboxylates and perfluorooctane sulfonate via consumption of meat, fish, fast foods, and food items prepared in their packaging. J. Agric. Food Chem. 2007, 55 (8), 3203-3210.

(61) Kärrman, A.; Harada, K. H.; Inoue, K.; Takasuga, T.; Ohi, E.; Koizumi, A. Relationship between dietary exposure and serum perfluorochemical (PFC) levels-A case study. Environ. Int. 2009, 35 (4), $712-717$.

(62) Heo, J.-J.; Lee, J.-W.; Kim, S.-K.; Oh, J.-E. Foodstuff analyses show that seafood and water are major perfluoroalkyl acids (PFAAs) sources to humans in Korea. J. Hazard. Mater. 2014, 279, 402-409.

(63) Fu, J.; Gao, Y.; Wang, T.; Liang, Y.; Zhang, A.; Wang, Y.; Jiang, G. Elevated levels of perfluoroalkyl acids in family members of occupationally exposed workers: the importance of dust transfer. Sci. Rep. 2015, 5, 9313.

(64) Banzhaf, S.; Filipovic, M.; Lewis, J.; Sparrenbom, C. J.; Barthel, R. A review of contamination of surface-, ground-, and drinking water in Sweden by perfluoroalkyl and polyfluoroalkyl substances (PFASs). Ambio 2017, 46, 335-346.

(65) Vestergren, R.; Cousins, I. T. Human dietary exposure to perand poly-fluoroalkyl substances (PFASs). In Persistent Organic Pollutants and Toxic Metals in Foods; Rose, M., Fernandes, A., Eds; 2013; 279-307.

(66) Benford, D.; Boer; de, J.; Carere, A.; Domenico; di, A.; Johansson, N.; Schrenk, D.; Schoeters, G.; De Voogt, P.; Dellatte, E. Opinion of the scientific panel on contaminants in the food chain on perfluorooctane sulfonate (PFOS), perfluorooctanoicacid (PFOA) and their salts. EFSA J. 2008, 653, 1-131.

(67) COT Statement on the Tolerable Daily Intake for Perflourooctane Sulfonate; COT Statemnet; Committee on Toxicity of Chemicals in Food; Consumer Products and the Environment (COT): 2006.

(68) Knepper, T. P., Lange, F. T., Eds. Polyfluorinated chemicals and transformation products. In the Handbook of Environmental Chemistry; 2012; $1-174$.

(69) Drinking Water Health Advisory for Perfluorooctanoic Acid (PFOA), EPA 822-R-16-005; United States Environmental Protection Agency: Washington, DC, 2016.

(70) Borg, D.; Lund, B.-O.; Lindquist, N.-G.; Håkansson, H. Cumulative health risk assessment of 17 perfluoroalkylated and polyfluoroalkylated substances (PFASs) in the Swedish population. Environ. Int. 2013, 59, 112-123. 